\title{
A relapse of pemphigus vulgaris in pemphigus herpetiformis or a phenotypic "switch"
}

\section{Siham Belmourida, Meriame Meziane, Nadia Ismaili, Laila Benzekri, Badreddine Hassam, Karima Senouci}

Department of Dermatology-Venereology, Mohammed V University, IBN Sina Hospital, Rabat, Morocco

Corresponding author: Siham Belmourida, MD, E-mail: belmouridasiham2017@gmail.com

Sir,

Pemphigus herpetiformis (PH) was originally described by Jablonska et al. in 1975. Clinically, PH presents itself as a herpetiform dermatitis with immunopathological characteristics of pemphigus $[1,2]$.

We report an exceptional case of typical pemphigus vulgaris (PV) relapsing after 36 years in $\mathrm{PH}$.

A 65-year-old patient, followed for PV for 36 years and treated with corticosteroid therapy with a remission for more than thirty years, consulted for pruriginous lesions evolving for the previous eight months. A dermatological examination revealed urticariform pruriginous ring lesions surmounted by small peripheral vesicles spread throughout the body (Fig. 1), sparing the mucous membranes, and without Nikolsky's sign. After two non-specific skin biopsies, the histological examination revealed an intraepidermal bubble with acantholytic cells and eosinophilic spongiosis (Figs. 2a and 2b). Direct immunofluorescence confirmed the diagnosis of pemphigus and indirect immunofluorescence was at the upper limit. The diagnosis of a PV relapse in PH was retained and a dapsone-based treatment was initiated at a dose of $150 \mathrm{mg} /$ day and stopped seven days later when met with hemolytic anemia. Oral corticosteroid therapy involving prednisone at a dose of $1 \mathrm{mg} / \mathrm{kg} / \mathrm{day}$ was initiated but, given the persistence of the pruritus, the decision was to combine methotrexate at a dose of $12.5 \mathrm{mg} /$ week. A good evolution and a decline within eight months were observed.

An improved pruritus and the disappearance of the skin lesions were achieved after one month of treatment.
PV and PH are two different anatomical and clinical entities of the autoimmune disease pemphigus, with distinct clinical, histopathological, and immunopathological characteristics $[1,2]$.

Our observation documents a complete phenotypic "switch" of pemphigus with a transition from PV to PH both clinically, histologically, and immunologically. Several rare cases of PV switching to superficial pemphigus (SP) ("phenotypic switch") have, since 1991, been reported, with a higher frequency this direction than otherwise; the transition period varies from six months to twenty years [3].

To the best of our knowledge, no case has been described of a progression from PV to PH. Having observed one firsthand, we are first to describe the case of a complete phenotypic switch from PV to PH. The mechanism of such a transition remains poorly understood and is often observed during a relapse. Some authors suggest that the effect of immunosuppressants on the desmoglein DSG3 more marked than on DSGl could explain the relapse of PS in PH $[3,4]$.

Future studies on the immunological factors and predictors of PV relapses after the discontinuation of treatment would be useful to better understand the mechanisms of a relapse in pemphigus, with or without a phenotypic transition.

\section{Consent}

The examination of the patient was conducted according to the principles of the Declaration of Helsinki.

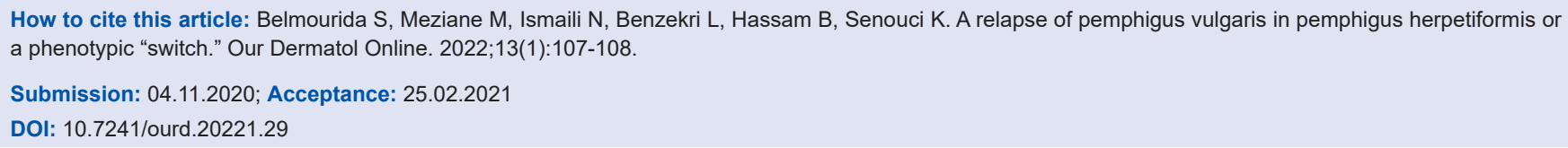




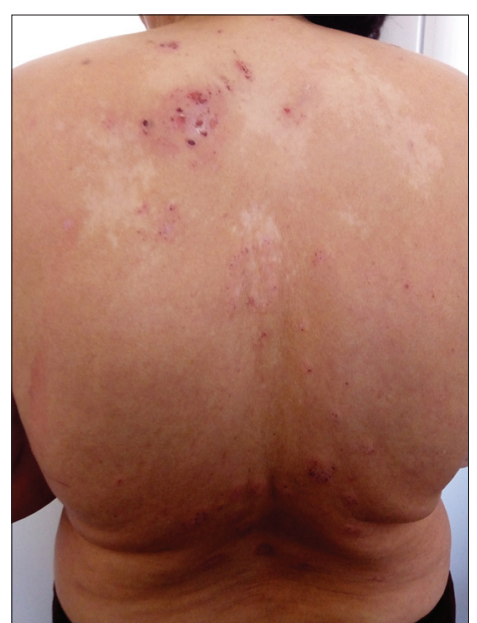

Figure 1: Urticariform pruriginous ring lesions surmounted by small peripheral vesicles.

The authors certify that they have obtained all appropriate patient consent forms, in which the patients gave their consent for images and other clinical information to be included in the journal. The patients understand that their names and initials will not be published and due effort will be made to conceal their identity, but that anonymity cannot be guaranteed.

\section{REFERENCES}

1. Jablonska S, Chorzelski T, Beutner E, Chorzelska J. Herpetiform pemphigus, a variable pattern of pemphigus. Int J Dermatol. 1975;14:353-9.

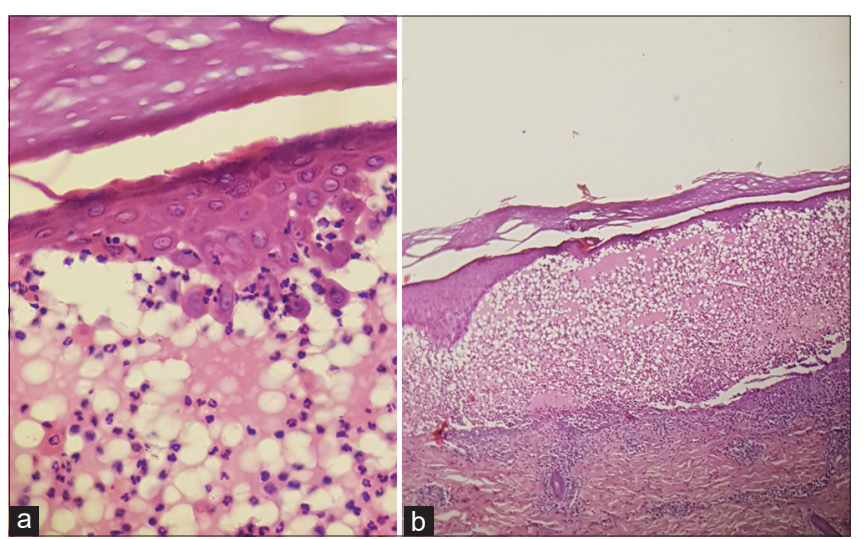

Figure 2: (a-b) Histological image showing an intraepidermal bubble with acantholytic cells and eosinophilic spongiosis.

2. Micali G, Musumeci ML, Nasca MR. Epidemiologic analysis and clinical course of 84 consecutive cases of pemphigus in eastern Sicily. Int J Dermatol. 1998;37:197-200.

3. Lévy-Sitbon C, Reguiaï Z, Durlach A, Goeldel AL, Grange. F, Bernard P. Transition from pemphigus vulgaris to pemphigus foliaceus: A case report. Ann Dermatol Venereol. 2013;140:788-92.

4. Abreu Velez AM, Upegui-Zapata YA, Valencia-Yepes CA, UpeguiQuiceño E, Yi H, Vargas Florez A, et al. A new variant of endemic pemphigus foliaceus in Colombia South America. Our Dermatol Online. 2020;11:284-99.

Copyright by Siham Belmourida, et al. This is an open access article distributed under the terms of the Creative Commons Attribution License, which permits unrestricted use, distribution, and reproduction in any medium, provided the original author and source are credited. Source of Support: Nil, Conflict of Interest: None declared. 